\title{
Psychiatric and behavioural manifestations of Huntington's disease
}

\author{
Sameer Jauhar \& Stuart Ritchie
}

Sameer Jauhar is a specialty registrar in general adult psychiatry in the West of Scotland Training Scheme. His interests are in epidemiology, public health and medical education, and his research interests are in the fields of addiction and psychosis. Stuart Ritchie is a consultant old age psychiatrist based in Leverndale Hospital, Glasgow. He provides generic old age psychiatric services to a catchment area in south Glasgow, old age psychiatric liaison services to the Southern General Hospital and has a special interest in Huntington's disease. He has been running a Huntington's disease management clinic at the Department of Medical Genetics, Yorkhill Hospital for 11 years. Correspondence Sameer Jauhar, Department of Psychiatry, Hairmyres Hospital, Eaglesham Road, East Kilbride G75 8RG, UK. Email: sameerjauhar@yahoo.co.uk

\begin{abstract}
SUMMARY
Patients with Huntington's disease can be a challenge to psychiatrists and to psychiatric services. We present a patient whose case history illustrates this and underlines the need for prompt and adequate treatment of psychiatric problems in this population. We discuss the psychiatric and behavioural manifestations of the illness, the treatments available and the role of psychiatric services in providing care for people with Huntington's disease.
\end{abstract}

\section{DECLARATION OF INTEREST}

None.

Huntington's disease is an autosomal-dominant neurodegenerative disorder characterised by a triad of motor, cognitive and psychiatric symptoms (Box 1). It is the most common single-gene neurodegenerative disorder and has a prevalence of 4-10 per 100000 of the population, with regional variations (Paulsen 2001). The psychiatric manifestations cause significant morbidity and encompass the full spectrum of psychiatric illness. We present a case from our clinical practice that illustrates the role of psychiatric assessment and

BOX 1 Symptoms of Huntington's disease

$\begin{array}{ll}\text { Motor symptoms } & \text { Psychiatric symptoms } \\ \text { - Choreiform movements } & \text { • Anxiety } \\ \text { - Falling, stumbling, difficulty in walking } & \text { • Depressive symptoms } \\ \text { - Clumsiness, loss of balance/coordination } & \text { • Possible manic symptoms } \\ \text { - Decreased volitional movement } & \text { - Delusions } \\ \text { - Difficulty eating, speaking and swallowing } & \text { - Hallucinations } \\ \begin{array}{l}\text { Cognitive symptoms } \\ \text { - Memory impairment }\end{array} & \text { - Lack of motivation/energy } \\ \text { - Decreased concentration } & \text { - Suicidal thoughts } \\ \text { - Difficulty in planning, organising and } & \\ \text { reasoning (executive symptoms) } & \\ \text { - Behavioural change (irritability, apathy) } & \end{array}$

service provision. Although the patient has given permission to publish, details have been changed to protect her privacy.

\section{Case study}

A homeless single mother in her 20s with maternal history of Huntington's disease presented to casualty following an attempt to hang herself.

She had first presented to psychiatric services at the age of 13 . She was referred by the social services department following a deteriorating relationship with her mother, in addition to stealing and truancy. Her mother had been diagnosed the previous year with the clinical onset of Huntington's disease and was being treated for depression. The patient had taken an impulsive overdose of antibiotics having argued with her mother and was subsequently assessed by a psychiatry trainee, who elicited no psychiatric symptoms at that time. At initial interview she did not want to discuss matters further and was referred back to social services.

The patient had left school early to look after her mother. She had gained no qualifications and was unable to find paid employment. She did not appear to maintain any significant relationships. She became pregnant aged 17, her son being taken into the care of her sister due to the patient's chaotic lifestyle and alcohol misuse. She had recently been evicted from a woman's shelter following a drunken assault on another resident.

She was seen at the age of 19 after an overdose, having had a drunken argument with her sister. She described recent suicidal thoughts, irritability and problems with her memory, and on account of continued suicidal ideation was admitted to a psychiatric ward. She described feeling low in her mood since the birth of her son 2 years previously. Alcohol intake was on a daily basis, with evidence of compulsion and psychological craving, although nothing to suggest physical dependence. She had assault charges pending at that time, all in the context of intoxication. After a 1-week admission she was given a diagnosis of borderline personality disorder and discharged without psychotropic 
medication, with follow-up from addiction services. She was re-referred by her general practitioner (GP) complaining of depression, although at that time desisted from follow-up. It was during this period that she requested predictive Huntington's disease gene testing, although she did not attend for initial counselling and did not receive testing.

On admission in 2007 following her attempted hanging, she described hearing the 'Devil's voice' commenting about her in a derogatory fashion during the previous week. She described this voice controlling her body, and complained of 'spiders' crawling over her at night. Her mood was subjectively low and she described sleep disturbance, denying other affective symptoms. She denied recent illicit drug use or alcohol excess. She spoke about the recent suicide of her ex-partner while in prison, and was admitted to a psychiatric ward for further assessment.

\section{Family history}

The patient's mother had developed Huntington's disease with early cognitive impairment in her early 40s. She died during the writing of this article. Her two elder sisters have had no prior psychiatric contact, and there is a poor relationship between them and the patient. A maternal uncle has Huntington's disease, and has received psychiatric treatment for substance misuse and psychosis. He is a long-term resident in a nursing home.

\section{Examination}

On examination she was noted to be responding to external stimuli and was irritable in manner towards nursing staff. She was periodically unresponsive, staring blankly into space. Her oral intake was poor and she was clinically dehydrated. Her mood was felt to be depressed, with a flattened affective response. She described clear passivity phenomena relating to the Devil, and experienced somatic hallucinations of spiders under her skin, particularly at night. There was no evidence of thought disorder. No comment was made on her cognitive functioning.

\section{Diagnosis and treatment}

She was diagnosed with a psychotic illness and initially treated with risperidone long-acting injection, followed by oral risperidone. There was an improvement in her mental state and she was discharged after 5 weeks to homeless hostel accommodation. Following discharge she described deterioration in her mental state, with increased frequency and intensity of auditory and tactile hallucinations, possibly due to poor medication concordance. A few weeks after discharge she took an impulsive overdose of paracetamol tablets, necessitating readmission. She described clear feelings of worthlessness, anergia, sleep disturbance and subjective low mood. Her affective response was decreased. A depressive episode was diagnosed and citalopram initiated. She responded well, with a decrease in Montgomery-Åsberg Depression Rating Scale (MADRS; Montgomery 1979) score from 34 to 17. Although she continued to describe ongoing auditory hallucinations, she reported their intensity to be reduced. On cognitive testing using the Addenbrooke's Cognitive Examination Revised (ACE-R; Mioshi 2006) she scored 65/100 (normal range $>82$ ), with impaired executive functioning, recall and working memory.

In light of her psychiatric symptoms and family history of Huntington's disease, predictive testing was discussed. At her request she was referred to the genetics department. There was no clear evidence of movement disorder at this time. The geneticist commented on the marked change in her personality, having seen her accompany her mother to the clinic in the past. Gene testing revealed a CAG expansion of 54 repeats (normal range 8-26; intermediate 27-35; reduced penetrance 36-39; 40 or more, full penetrance of Huntington's disease; Tibben 2007).

She continued to experience chronic auditory hallucinations, although described the intensity as decreasing. She agreed to a trial of aripiprazole, although this was accompanied by significant worsening of her psychotic symptoms, possible akathisia and associated suicidality. Resumption of risperidone, at the prior dose, resulted in her mental state returning to its previous level. She was discharged to supported accommodation after 1 year in hospital.

\section{Psychiatric disorders associated with Huntington's disease}

Although psychiatric disorder is not an inevitable consequence of Huntington's disease, the prevalence of psychiatric symptoms is significantly higher than in the general population; therefore there is a need for awareness of psychiatric morbidity at primary and secondary care levels. History-taking should include a corroborative history from caregivers. The importance of early detection is exemplified by the case study, in which the patient presented with behavioural features and psychiatric symptoms of Huntington's disease years before diagnosis. By the time of diagnosis, she was estranged from her family, had given birth to a son (who has a 50\% chance of inheriting the disease) and had endured distressing psychiatric symptoms for a significant period of time, without adequate treatment. 
The care of patients with Huntington's disease is predominantly multidisciplinary and should be conceptualised in a manner similar to that for any patient with a severe and enduring mental illness. Although psychiatric and cognitive symptoms may pre-date the motor symptoms, a broad consensus exists that a definite diagnosis of Huntington's disease cannot be made until the emergence of the specific motor disorder.

\section{Depression}

In his original description, George Huntington described 'a tendency to insanity and suicide' (Huntington 1872). The prevalence of mood disorder in those with Huntington's disease has been estimated to be as high as 38\%, with $22 \%$ meeting DSM criteria for major depression (Folstein 1983). The aetiology of depression is thought to be twofold (Paulsen 2001), and linked to psychosocial and biological factors. There is the adjustment to a terminal illness and the disability and grief associated with this. Depression has also been linked to neuronal loss in the medial caudate, with the connection between this and orbitofrontal and prefrontal cortices, illustrated by positron emission tomography findings of decreased glucose metabolism in these areas (Lauterbach 1998). Depression may present with agitation, change in personality or social withdrawal. It is worth noting that some of the symptoms of depression can be difficult to detect in Huntington's disease, given the effects of the illness on factors such as appetite, sleep, concentration and motivation.

Although there are no randomised controlled trials of antidepressant therapy in Huntington's disease, principles of treatment are broadly similar to those of depression in general, with case reports suggesting efficacy of selective serotonin reuptake inhibitors (SSRIs), tricyclic antidepressants, monoamine oxidase inhibitors and mirtazapine (Rosenblatt 1999). Tricyclics should be used with caution in light of possible anticholinergic-induced cognitive decline and falls. Monoamine oxidase inhibitors may worsen choreiform movements. As in populations without Huntington's disease, electroconvulsive therapy would remain a therapeutic option in severe depression. There is little existing evidence on the efficacy of cognitivebehavioural therapy or other psychotherapies in patients with Huntington's disease and depression, and pre-existing cognitive impairment, particularly executive dysfunction, should be borne in mind.

\section{Suicide and self-harm}

The suicide rate for patients with Huntington's disease may be four to six times higher than that for the general population, increasing in those over the age of 50. Suicide rates have been reported as being as high as $12.7 \%$ (Schoenfeld 1984) and suicide is the third most common cause of death in Huntington's disease, after pneumonia and cardiovascular disease. The aetiology of suicide in Huntington's disease is multifactorial, contributory factors including poor adjustment to diagnosis, denial of diagnosis, impulsivity, depression and irritability.

Self-harm has not been documented clearly in the literature; clinical experience suggests that it occurs in the context of behaviour changes associated with Huntington's disease (see below), and psychiatric morbidity (illustrated by the case study). Risk factors for repeated self-harm are similar to those seen in self-harm in general.

\section{Psychosis}

An increased frequency of psychosis has been found in Huntington's disease, a review of the literature estimating a schizophrenia-like psychosis occurring in 3-6\% of patients (Naarding 2001). Psychotic symptoms in the broader sense occur in about $3-11 \%$ of patients and are usually associated with increasing cognitive impairment (van Duijn 2007), as illustrated in the case study. Early age at onset and family history of Huntington's disease-associated psychosis are both risk factors. Positive symptoms tend to become less overt as the disease (and cognitive impairment) progresses (Mendez 1994). Positive symptoms do not follow a characteristic course. They can be difficult to differentiate from a non-organic psychosis on initial presentation, but they can be distinguished from delirium by the lack of fluctuation in clinical course (as seen in the case study).

The underlying cause of psychosis in Huntington's disease is not clearly understood and various theories have been put forward, including direct damage to the caudate, an excess hyperdopaminergic state (as a result of neuronal loss) and hypofrontality. As illustrated by the case study, psychosis may pre-date motor manifestations of the disease, and have significant effects on quality of life. Atypical antipsychotics with a low incidence of extrapyramidal sideeffects are preferred, to avoid exacerbation of the bradykinesia inherent in the illness.

\section{Mania}

Although occurring to a substantially lesser degree than unipolar illness, there is a suggestion that up to $4.8 \%$ of patients with Huntington's disease develop bipolar affective disorder (Mendez 1994). Hypomania or manic episodes were found in up to 
$10 \%$ of the cohort in a study by Folstein $\&$ Folstein (1983). Nevertheless, in the 10 years of treating Huntington's disease in the west of Scotland, we have only seen one case of true mania, and this was in a pre-symptomatic gene-positive patient.

As in all psychiatric manifestations of Huntington's disease, evidence for treatment is scarce. Lithium may not be first-line therapy in this patient group, and both carbamazepine and sodium valproate have been suggested. In keeping with current practice, the use of antipsychotic medication may be indicated (Rosenblatt 1999).

\section{Dementia/cognitive changes}

Cognitive deterioration is an invariable feature and presents early in the course of the illness. This is seen in the case study, where the patient presented with these features years before they were recognised or the diagnosis considered. Pre-symptomatic cognitive changes are well recognised, although there is relative preservation of cognitive function when the illness presents in later life. The cognitive disorder of Huntington's disease is generally thought to be a subcortical syndrome and it lacks the typical features found in cortical dementias such as Alzheimer's disease (e.g. dysphasia, dyspraxia, amnesia; Table 1). Problems with executive functioning are the most prominent and include difficulties with organisation, reasoning, planning, judgement, impulse control and decision-making.

Many of the changes in behaviour associated with Huntington's disease arise from the cognitive deficits, and these contribute more to the loss of independence and to functional disability than does the primary motor disorder. Communication is increasingly disrupted by impairment in the motor production of speech. Huntington's disease does not cause primary language dysfunction such as the word-finding difficulties, paraphasic errors or receptive disorders that are typically associated with Alzheimer's disease and other cortical dementias. Care should be taken to distinguish language function, which is relatively preserved, from impairment in the motor production of speech. In the latter stages of the illness most patients become unintelligible, although this does not signify a lack of comprehension and therefore clinicians should maintain communication with patients, even in the absence of a response.

Although most patients will complain of memory problems, they do not have amnesia in the classical sense, and are able to learn, retain and retrieve new material given the appropriate circumstances. Impairment of attention and concentration along with organisational failure gives rise to subjective
TABLE 1 Comparison of cognitive deficit in Alzheimer's and Huntington's dementia

\begin{tabular}{|lll|}
\hline Clinical feature & Alzheimer's dementia & Huntington's dementia \\
\hline Onset & Gradual & Gradual \\
\hline Cortical/subcortical & Cortical & Subcortical \\
\hline Executive function & $\begin{array}{l}\text { Impairment correlates with } \\
\text { general cognitive decline }\end{array}$ & $\begin{array}{l}\text { Early and significant impairment, } \\
\text { disproportionate to other cognitive } \\
\text { domains }\end{array}$ \\
\hline Language function & Significantly affected & $\begin{array}{l}\text { Relatively preserved, until later } \\
\text { stages }\end{array}$ \\
\hline Visuospatial function & Significantly affected & Relatively preserved \\
\hline Semantic memory & Significantly affected & Relatively preserved \\
\hline
\end{tabular}

memory problems. Semantic memory is relatively preserved in Huntington's disease.

Even at an early stage, executive dysfunction is apparent and can be elicited by careful historytaking. An example in the case presented above is the patient's impulsivity and what appears to be decreased abstract reasoning when she presented at the age of 19. Detailed neuropsychological testing is useful but not always necessary, as these changes can be detected using easily administered bedside tests of executive functioning, for example verbal fluency and the Stroop Interference Test (Stroop 1935).

Pertinently, the cognitive changes and lack of insight are factors that contribute to the characteristic behaviour disorder of Huntington's disease (specifically apathy and irritability, see below). Lack of volition and apathy are usually present and without an external structure, patients often withdraw, with a failure to maintain daily routines. There is no evidence to suggest an improvement in cognitive functioning with acetylcholinesterase inhibitors (Cubo 2006).

\section{Behavioural changes}

Craufurd et al (2001) recorded high prevalence of a range of behavioural problems in Huntington's disease and identify three clusters of symptoms on factor analysis - apathy, irritability and depression. The apathy factor was highly correlated with duration of illness, although no such relationship was identified with the other factors (Fig. 1). Impulsivity, irritability and verbal outbursts are a common feature of the illness, causing interpersonal difficulties, figures for irritability ranging from 15 to $60 \%$ depending on definition. Before considering pharmacotherapy it is essential to explore the context of irritability, with an attempt to identify possible antecedents. Although there is a dearth of evidence on this topic, clinical experience has shown that SSRIs, mood stabilisers and low-dose antipsychotics can be of 


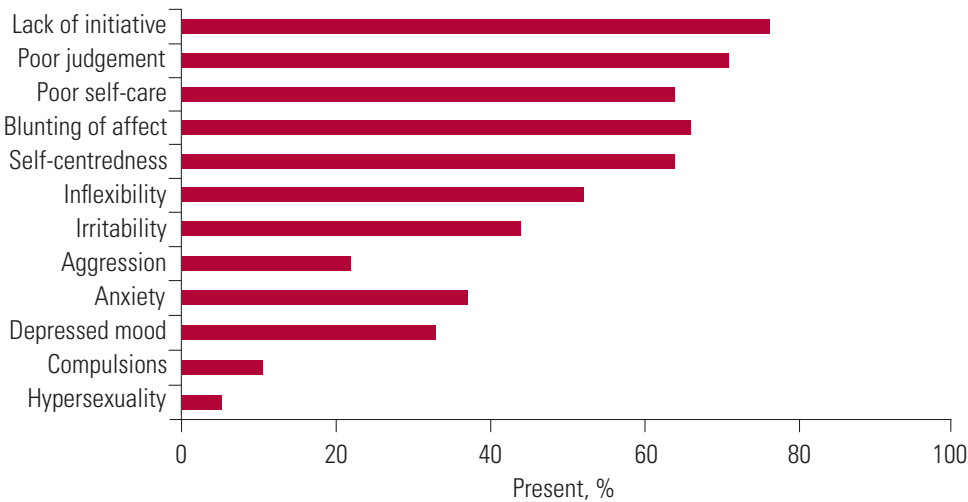

Frequency of behavioural changes in Huntington's disease (Craufurd 2002: p. 84. Reproduced with permission of Oxford University Press).

value, although none are licensed for Huntington's disease-related aggression (Rosenblatt 1999). Although hypersexuality is commonly perceived as being present, the converse is true, sexual dysfunction and loss of libido being common in Huntington's disease (Craufurd 2002).

\section{Anxiety disorders}

Anxiety disorders have been studied relatively less well than other psychiatric symptoms of the disease. Although an early study described anxiety as the most common prodromal symptom, occurring in $12 \%$ of 102 patients (Dewhurst 1970), it is the symptoms of anxiety that are more often seen rather than a primary anxiety disorder. This group of symptoms is likely to be secondary to underlying cognitive changes and should be considered as a separate neuropsychiatric behaviour disorder. Treatment of anxiety symptoms or disorder is broadly similar to that of patients without Huntington's disease, although benzodiazepines should be monitored carefully in light of the increased incidence of falls, effects on cognition and impaired judgement, and increased risk of precipitating delirium.

\section{Obsessive and compulsive symptoms}

Although not as prevalent as in other diseases of the basal ganglia (e.g. Tourette syndrome), there are numerous case reports of obsessive-compulsive disorder (OCD) in Huntington's disease, and some have suggested a familial predisposition. A significant percentage of patients with the disease have obsessional symptoms, without meeting the formal criteria for OCD, obsessions being seen more commonly than compulsions (studies place symptom prevalence between 10 and 52\%; van Duijn 2007). Repetitive verbal utterances and behaviours are common, and are associated with executive dysfunction rather than secondary features of OCD. Although evidence is scarce, general guidelines for treatment of OCD should be followed, and cognitive function allowed for if psychological therapy is introduced.

\section{Substance use disorders}

Evidence is equivocal on the prevalence of substance use disorders in this population, a recent study (Ehret 2007) showing increased use of alcohol and cigarette smoking as psychiatric symptoms worsen, and increased use in those with early-onset Huntington's disease. Screening and treatment of comorbid substance misuse is appropriate. Substance misuse can accentuate the behavioural change seen in the illness, as demonstrated in the case study. As for other psychiatric manifestations, cognitive (especially executive) changes should be acknowledged when initiating treatment for substance use disorders, both in terms of social environment (and possible cues) and psychotherapy.

\section{Predictive testing}

As illustrated in the case study, predictive testing for the Huntington's gene is provided by clinical genetics departments. Genetic counselling preand post-testing is an essential component of the process. In the case study, staff from the clinical genetics department were supportive during the predictive testing process, especially considering the patient's mental state and in-patient status.

Predictive testing in Huntington's disease is a patient-led process and default from genetic counselling is not uncommon, reflecting the patient's wish to withdraw from testing.

\section{Presentation}

The large majority of patients presenting with Huntington's disease have a known family history of the illness. Many of these patients know their diagnosis without reference to medical services, and will present at varying stages of illness depending on their particular symptom profile. This group do not present to neurology, and the GP may refer them to genetics, psychiatry or rehabilitation medicine.

There continue to be small but significant numbers of people presenting late in the illness, having never come to the attention of medical services. This may be associated with an absent family history or lack of insight. Where there is no family history, presentation tends to be to neurological services, which excel with regard to appropriate investigation and neuroimaging. Diagnosis normally follows quickly, as does referral to any local services available for patients with Huntington's disease. 
There is often considerably greater distress and emotional upset in these patients and at-risk family members compared with patients who have grown up with an affected parent and/or other family member. In the latter circumstances, at-risk family members are more likely to seek predictive gene testing.

\section{Diagnosis}

Diagnosis is straightforward if a patient with a known family history of Huntington's disease presents with the typical neurological movement disorder. Gene testing in these circumstances is not normally required provided there is laboratory confirmation of the Huntington's disease gene in the family. The diagnosis of the disease in a patient without a family history is often delayed, and gene testing is essential. Nonetheless, it does not give a clinical diagnosis and must be combined with a clinical history and physical examination. Psychiatric presentation prior to the involuntary motor disorder stage is not uncommon, but as already mentioned there needs to be evidence of the involuntary movement disorder before a clinical diagnosis of Huntington's disease can be made. There is good evidence of early cognitive changes occurring before the onset of the involuntary motor disorder, as stated above (Lauterbach 1998).

\section{Capacity}

Capacity is a complex issue for patients and doctors alike. As described earlier, the cognitive deficits of the disease reduce patients' ability to make competent decisions based on all information available to them. Patients (according to the stage of the illness) tend to make decisions impulsively in light of immediate need, with reduced awareness of the alternatives or properly thinking through the consequences. With appropriate support, capacity for day-to-day decision-making can be maintained late into the illness, but for complex issues capacity is impaired relatively early. This is evidenced by the early occupational failure that typically occurs, particularly in patients with professional occupations.

The outcome and certain particular clinical issues of Huntington's disease are predictable and clinicians should encourage patients to discuss their wishes regarding future medical interventions while capacity is retained. Living wills and advance directives are suitable vehicles in which to record and communicate patients' wishes. The issue of parenteral feeding tubes in a patient without capacity often taxes families and clinicians, and if the patient's past wishes are not known, strong views are often expressed that may reflect personal values rather than an objective assessment of the benefit of treatment. Patients should be involved in the treatment of their illness as much as possible and any intervention should take account of past and present wishes as far as possible (Simpson 2006).

\section{Management}

Psychiatrists are well placed to play a central role in the care of people with Huntington's disease, owing to the high prevalence of psychiatric and behavioural symptoms. Patients often fail to engage with services for a variety of reasons, including patient (lack of insight and denial of illness) and service (the patient having to fit into existing and poorly fitting services) factors. Services may fail to recognise declining cognition and capacity, seeing the patient's lifestyle as a choice rather than a consequence of their illness. In the face of mounting service demands, community mental health teams (CMHTs) may deny responsibility for the patient's care, citing an absence of 'hard' psychiatric symptoms (e.g. psychotic and affective symptoms).

Many people with Huntington's disease display significant neuropsychiatric symptoms, but with coordinated multidisciplinary input and good home support these difficulties can be managed. There are, however, significant numbers of patients with complex needs who come to the attention of psychiatric services. These patients often display a range of psychiatric symptoms, for example hallucinations or low mood, which coexist with behavioural change and symptoms such as apathy, irritability and aggression. There may be failure of family or formal services to cope with their care at home. Lack of insight is often a feature, with an associated avoidance of services.

This group should be thought of in the same way as people with severe and enduring mental illness (e.g. chronic schizophrenia). Services which provide active and assertive outreach in the community, with an emphasis on social and housing support, are preferable. These patients benefit from assistance navigating through social and voluntary services, and advocacy on their behalf is essential. ${ }^{\dagger}$ Community psychiatric teams should maintain contact with the patients and need to remember that Huntington's disease is a long-term illness, akin to chronic schizophrenia. There is a central role for coordination and liaison. As with all progressive illnesses, problems need to be anticipated, with regular education and advice to both the patient and their carers. Keeping the patient informed and involved in their care is a central tenet of their treatment.
${ }^{\dagger}$ For a discussion of mental health advocacy see Harrison T, Davis R (2009) Advocacy: time to communicate. Advances in Psychiatric Treatment 15: 57-64. Ed. 


\section{Treatment in the community}

Managing Huntington's disease in patients with such complex needs may be time consuming but highly rewarding. Treatment in the community follows the same principles of care as any severe and enduring mental illness, and involves thorough and regular assessment, use of the multidisciplinary team and good communication. Use of the care programme approach (where this is not a statutory requirement) may facilitate multidisciplinary working especially when involving social services, housing and other community services. The Huntington's Disease Association has branches in most areas and can be invaluable, providing a range of services, including specialist workers, advocacy and youth services in addition to advice on specific needs relating to, for example, housing or benefits.

\section{Admission to hospital}

It is our clinical experience that the majority of hospital admissions during the predominant course of the illness are to psychiatric services. A small number of patients with the disease, usually those with a background of failure to cope, require admission because of severe psychiatric illness or a breakdown or refusal of community services. Treatment of the psychiatric illness is generally straightforward and placement in hospital provides a secure and structured environment, addressing many of the neuropsychiatric behaviours that may have precipitated admission.

Cognitive deficits combined with neuropsychiatric symptoms often cause the greatest difficulties in behaviour, for example anergia, lack of initiative, blunted affect, egocentricity, constant demands, irritation and threatening behaviour. In-patient staff may make comments along the lines of "patient A shouldn't be here'. This feeling may arise from an absence of a hard 'psychiatric' diagnosis, perhaps reflecting a common perception that patients with Huntington's disease are not primarily 'psychiatric' in nature. There may be a failure to appreciate the organic aetiology of the behavioural disorder of the disease and the limited control the patient has over their symptoms. The patient may be labelled as 'lazy' or 'demanding', with subsequent alienation. In-patient staff often underestimate the importance of their role in providing a structured environment and the resultant benefits this has in containing the neuropsychiatric symptoms. The problem behaviours often resurface if discharge from hospital occurs without appropriately structured community services. Use of the Mental MCO answers
Health Act may be appropriate, and should follow the principles laid out in the legislation.

\section{Returning home and alternative housing}

By far the most satisfactory outcome for patients with complex needs arising from Huntington's disease is for an individual and tailored package of care in their own home, informed by a multidisciplinary assessment. Services can include home support care, befriending services and day care, in addition to support from Huntington's disease organisations, which can provide training to the various care providers. Regular reassessments are required, given the progressive and fluctuating nature of this illness.

If a return home is not appropriate, alternatives are limited, especially for those under 65 (the cut-off age for old age services). Many young and middle-aged patients are sent to nursing homes registered for the young physically disabled. People with Huntington's disease, particularly if not in the later stages of the illness, do not fit particularly well into this environment as these units tend to be weighted towards nursing care of physically disabled people.

Supported accommodation projects are often a better choice as long as appropriate supervision is available. This type of accommodation maintains a higher degree of independence and can provide a better quality of life. Both psychiatric services and the organisations providing the accommodation may resist the placement of people with Huntington's disease in supported accommodation. Once again, this may be due to the combined difficulties of the patients' declining physical health and psychiatric illness. The neuropsychiatric symptoms, as mentioned above, and patients' unwillingness to engage may also cause difficulties.

Supported accommodation projects, with the availability of 24-hour care staff, can be very successful in maintaining patients with Huntington's disease in the community. These projects impose an external structure within which the patient can develop a degree of independence. In early and mid-stage Huntington's disease, this is certainly preferable to nursing home care.

\section{Service provision}

The expertise of local CMHTs in maintaining contact with those who have severe and enduring mental illness, using active outreach to support those reluctant to engage, is highly appropriate for some patients with Huntington's disease and should be harnessed. Generic community teams often feel that they lack the necessary skills owing to their lack of experience with and knowledge of the illness. Specialist teams obviously have greater experience and knowledge about the illness but tend to be small in size and have limited resources. 
Health boards are generally reluctant to provide diagnosis-specific services, preferring to increase psychiatric support to people with Huntington's disease through generic community teams.

There are many specialist Huntington's disease services across the UK and most urban areas now have some access to these. These services are not uniformly designed or specialist led, and may be based within psychiatric, genetic, rehabilitation or neurological services.

\section{Conclusions}

Although the neuropsychiatric symptoms of Huntington's disease are complex and disabling, prompt diagnosis and treatment of psychiatric morbidity offer significant benefits to patients. We advocate that specialist Huntington's disease services be based within generic psychiatric services and that a good working relationship be fostered between generic CMHTs and these specialist services. Joint care, utilising both the specialist knowledge of a Huntington's disease team and the extensive experience of a CMHT in dealing with avoidant and reluctant patients will undoubtedly provide the optimal outcome.

\section{References}

Craufurd D, Thomson JC, Snowden JS (2001) Behavioural changes in Huntington Disease. Neuropsychiatry, Neuropsychology, and Behavioral Neurology 14: 219-26.

Craufurd D, Snowden J (2002) Neuropsychological and neuropsychiatric aspects of Huntington's disease. In Huntington's Disease (3rd edn) (eds P Harper, G Bates, L Jones): 62-94. Oxford University Press.

Cubo E, Shannon KM, Tracy D, et al (2006) Effect of donepezil on motor and cognitive function in Huntington disease. Neurology 10: 1268-71.

Dewhurst K, Oliver JE, McKnight AL (1970) Socio-psychiatric consequences of Huntington's disease. British Journal of Psychiatry 116: $255-8$.
Ehret JC, Day PS, Wiegand R, et al (2007) Huntington disease as a dual diagnosis disorder: data from the national research roster for Huntington disease patients and families. Drug and Alcohol Dependence 86: 283-6.

Folstein SE, Folstein MF (1983) Psychiatric features of Huntington's disease: recent approaches and findings. Psychiatric Development 1: 193-205.

Huntington G (1872) On chorea. Medical and Surgical Reports of Philadelphia 26: 17-21

Lauterbach EC, Cummings JL, Duffy JC, et al (1998) Neuropsychiatric correlates and treatment of lenticulostriatal diseases: a review of the literature and overview of research opportunities in Huntington's, Wilson's, and Fahr's diseases. Journal of Neuropsychiatry and Clinical Neurosciences 10: 249-66.

Mendez MF (1994) Huntington's disease: update and review of neuropsychiatric aspects. International Journal of Psychiatry in Medicine 24: $189-208$

Mioshi E, Dawson K, Mitchell J, et al (2006) The Addenbrooke's Cognitive Examination Revised (ACE-R): a brief cognitive test battery for dementia screening. International Journal of Geriatric Psychiatry 21: 1078-85.

Montgomery SA, Åsberg M (1979) A new depression scale designed to be sensitive to change. British Journal of Psychiatry 134: 382-9.

Naarding $P$ (2001) Huntington's disease: a review of the literature on prevalence and treatment of neuro-psychiatric phenomena. European Psychiatry 16: 1639-45.

Paulsen JS, Ready RE, Hamilton JM, et al (2001) Neuropsychiatric aspects of Huntington's disease. Journal of Neurology, Neurosurgery and Psychiatry 71: 310-34.

Rosenblatt A, Ranen NG, Nance MA, et al (1999) A Physician's Guide to the Management of Huntington's Disease. Huntington's Disease Society of America.

Schoenfeld M, Myers RH, Cupples LA, et al (1984) Increased rate of suicide among patients with Huntington's disease. Journal of Neurology, Neurosurgery and Psychiatry 47: 1283-7.

Simpson SA (2006) Late stage care in Huntington's disease. Brain Research Bulletin 72: 179-81.

Stroop JR (1935) Studies of interference in serial verbal reactions. Journal of Experimental Psychology: General 18: 643-62.

Tibben A (2007) Predictive testing for Huntington's disease. Brain Research Bulletin 72: 165-71.

van Duijn E, Kingma EM, van der Mast RC (2007) Psychopathology in verified Huntington's disease gene carriers. Journal of Clinical Neuropsychiatry and Clinical Neuroscience 19: 441-8.

\begin{tabular}{|c|c|c|}
\hline MCOs & c poor judgement & c 4-10 per 100000 of the population \\
\hline Select the single best option for each question stem & $\mathrm{d}$ blunting of affect & d $4-10$ per 1000000 of the population \\
\hline 1 The lifetime risk of suicide in Huntington's & e hypersexuality. & e 4-10 per 10000000 of the population. \\
\hline $\begin{array}{l}\text { a less than } 2 \% \\
\text { b } 2-5 \%\end{array}$ & $\begin{array}{l}3 \text { The predominant cognitive impairment in } \\
\text { Huntington's disease is characterised by: }\end{array}$ & $\begin{array}{l}5 \text { By which mode is Huntington's disease } \\
\text { inherited? }\end{array}$ \\
\hline c $5-10 \%$ & a impaired executive function & a X-linked dominant \\
\hline d $10-15 \%$ & b dysphasia & b autosomal recessive \\
\hline e $15-20 \%$ & $\begin{array}{l}\text { c dyspraxia } \\
\text { d primary memory disorder }\end{array}$ & $\begin{array}{l}\text { c multifactorial inheritance } \\
\text { d autosomal dominant }\end{array}$ \\
\hline $\begin{array}{l}2 \text { The most common behaviour change in } \\
\text { Huntington's disease is: }\end{array}$ & e psychomotor retardation. & e X-linked recessive. \\
\hline $\begin{array}{l}\text { a irritability } \\
\text { b lack of initiative }\end{array}$ & $\begin{array}{l}4 \text { The prevalence of Huntington's disease is: } \\
\text { a } 4-10 \text { per } 1000 \text { of the population } \\
\text { b } 4-10 \text { per } 10000 \text { of the population }\end{array}$ & \\
\hline
\end{tabular}

